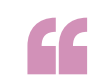

\section{a specific}

population of

neurons ... is

necessary and

sufficient to

induce aggression

in mice, and is

directly involved

in attack

behaviour. These

neurons are

suppressed during

mating

\title{
BEHAVIOUR
}

\section{All's fair in love and war}

Mating and aggressive behaviours in rodents have both been linked to the hypothalamus, but precisely which populations of neurons are involved in the encoding of these behaviours has remained unknown. A new paper shows that in male mice, the ventrolateral subdivision of the ventromedial hypothalamus (VMHvl) contains neurons that are active during fighting, and that some of these neurons are inactive or suppressed during mating. This partial mutual exclusivity suggests that competition exists between these two behaviours at cellular and circuit levels.

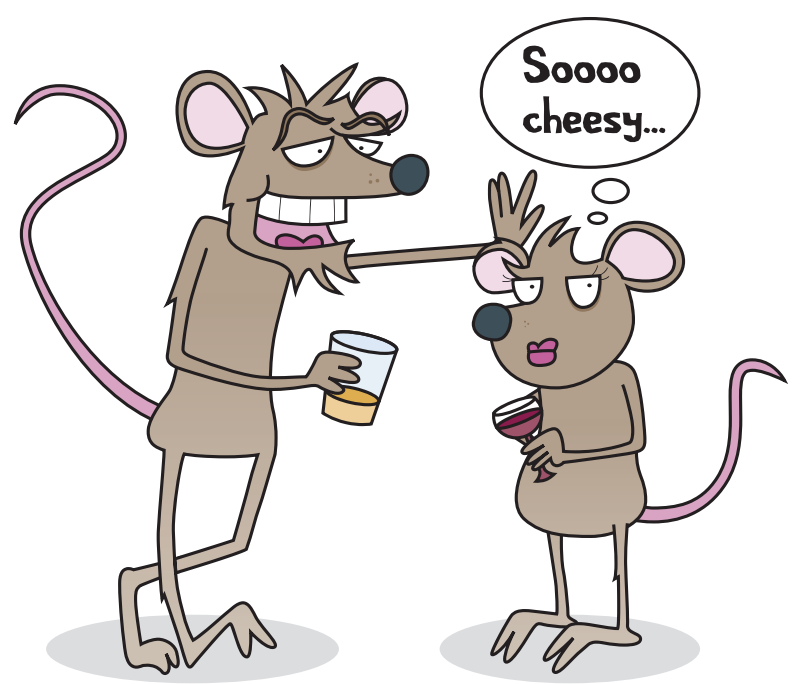

To test this hypothesis, Lin et al. examined mating behaviour and aggressive behaviour in male mice by presenting them with an unfamiliar female or male mouse, respectively, and then determined which cells in the VMHvl were involved in aggression and mating.

Using a form of fluorescent in situ hybridization to measure c-FOS expression, the authors showed that $20-30 \%$ of cells in the VMHvl were active during successive mating or aggressive events. This suggests that mating and aggression involve different (but overlapping) sets of neurons in this brain area.

Single-unit recordings in awake behaving animals subsequently showed that firing rates of individual neurons in the VMHvl increased during an aggressive encounter, and increased transiently during an encounter with a female, but decreased as mating progressed. Interestingly, the majority of neurons that were active during an aggressive encounter were selectively suppressed during mating.

Optogenetic stimulation of VMHvl neurons resulted in increased, and less discriminatory, aggression: most of the stimulated mice attacked female mice and even inanimate objects. Moreover, when subjected to an intruder mouse during mating, aggressive behaviour was strongly suppressed in these mice. Conversely, suppressing activity in the VMHvl reduced the latency to attack and the attack duration, supporting the notion that these neurons are involved in naturally occurring aggression.

These findings indicate that a specific population of neurons in the VMHvl is necessary and sufficient to induce aggression in mice, and is directly involved in attack behaviour. These neurons are suppressed during mating, and hence, one of their functions could be to suppress aggression during mating. They therefore constitute a neural correlate between mating and fighting and future work will involve investigating where this competition originates and how it is controlled.

Sian Lewis

ORIGINAL RESEARCH PAPER Lin, D. et al.

Functional identification of an aggression locus in the mouse hypothalamus. Nature 9 Feb 2011 (doi 10.1038/nature09736)

FURTHER READING Nelson, R. J. \& Trainor, B. C. Neural mechanisms of aggression. Nature Neurosci. 8, 536-546 (2007) 